\section{SOBRE A POSIÇĀO DO URETER NO HILO RENAL DE BOVINOS DA RAÇA HEREFORD}

ALTHEN TEIXEIRA FILHO
Professor Adjunto Universidade Federal de Pelotas

ANTONIO FERNANDES FILHO

Professor Titular

Faculdade de Medicina Veterinaria e Zootecria da USP

JOSE PEDUTI NETO

Professor Adjunto

Faculade de Medicina Veterinaria e

Zootecnia da USP

\section{INTRODUCAO}

7o realizar, anteriormente, pesquisa voltada para o número e disposicao de vasos arteriais e venosos no hilo renal de bovinos da raca Hereford, chamaramnos a atenço, variaças quanto a posiço, no local, de emergência do ureter. Sendo este aspecto, como puaemos verificar, notado de maneira superficial pelos tratadistas e tamuem năo considerado em outras pesquisas semelhantes realizadas em diversas especies e, mesmo em bovinos de diferentes origens, pareceu-nos interessante sistematizar seu estudo, com vista a caracteristica morfologica do animal examinado e, consequentemente, a enfoques de anatomia comparativa e racial.

\section{MATERIAL E METODO}

TEIXEIRA FILHO, A.; FERNANDES FILHO, ค.; PEDUTI NETO. J. Sobre a posiço do ureter no hilo renal de bovinos da raça Hereford. Rev. Fac. Med. Vet. Zootec. Univ. 5. Paulo, 25(2): 215-219, 1988.

RESUMO: Estudou-se o local de emergência do ureter no hilo renal de 30 bovinos da raca Hereford; para tanto, a regiao hilar foi dividida em quadrantes, assim designados: craniolateral, craniamedial, caudolaterat e caudomedial. Os resultados mostram variaças de localizaça, particularmente no rim esquerdo.

UNITERMOS: Anatomia, bovinos; Sistema urogenital; Rim; Ureter

\begin{abstract}
Para o estudo ora proposto, utilizamos, aos pares, rins de 30 bovinos da raca Hereford, 15 machos (Obs. 01 a 15) e 15 fêmeas (Obs. 16 a 30), adultos, abatidos no Matadouro Frigorifico Extremo Sul, sediado no municlpio de Pebotas, RS.

Os orgas eram retirados mantendose, alem do ureter, seus vasos unidos aos correspondentes segmentas da arteria aorta e da veia cava caudal; a seguir, eram fixados com soluca aquosa de formal a $10 \%$, je inlciopor injeço por. esses elementos e em seguida, por suamersao para, finalmente, serem dissecados.

A posiça do ureter é descrita como já feita para os elementos vasculares no trabalho citado inicialmente, obedecendo a divisao do hilo renal em quadrantes demarcados pelo tracado de 2 linhas, uma longitudinal, de polo a poloe, outra, transversal, perpendicular a primeira, interseccionadas no seu centro geometrico. Estes quadrantes sao designados como craniolateral, craniomedial, caudolatera! e caudomedial (Fig. 1).
\end{abstract}

\section{RESULTADOS}

Quanto ao local de emergência do ureter em bovinos da raca Hereford, estando a regigo hilar dividida nos quadrantes propostos, o material observado revelou-nos as disposicoes abaixo descritas, senda considerados, separadamente, o rim direito e o esquerdo, lembrando que, face aos dados obtidos, especificaremos apenas as observacies 
Sobre a posição do ureter no hilo renal de bovinos da raça Hereford.

correspondentes às peças que exibiram comportamento inusitado.

Rim Direito:

0 ureter, deste lado, emerge com maior frequência $(27$ vezes - 90,0\% \pm $5,5)$ pelo setor correspondente ao quadrante caudolateral. Pudemos, entretanto, localiza-lo tambem:

a) sobre a linha craniocaudal, entre os quadrantes caudolateral e caudomedial -2 vezes $(6,7 \% \pm 4,5$. Obs. 26 e 28);

b) sobre a linha mediolateral, entre as quadrantes craniolateral e caudolateral - 1 vez $(3,3 \% \pm 3,3$ 0bs. 10 Fig. 2)

Rim Esquerdo:

No rim esquerdo, o ureter aparece, na maioria dos casos (22 vezes-73,3\% 8, 1), tambem no quadrante caudolateral. Surpreendêmo-lo, porem, colocado ainda:

a) sobre a linha craniocaudal, entre os quadrantes caudolateral e caudomedial -4 vezes $(13,3 \% \pm 6,2$ abs $3,22,25,26)$;

b) sobre a inha mediolateral entre os quadrantes craniolateral e caudolateral - 2 vezes $(6,7 \% \pm 4,5$ Obs. $10,11)$

c) no quadrante craniolateral - 1 vez $(3,3 \% \pm 3,3$ Obs. 14 Fig. 3);

d) sobre a inha mediolateral entre os quadrantes craniomedial e caudomedial - 1 vez $(3,3 \% \pm 3,3$ Obs. 18).

Levando-se em conta as disposiças nao habituais, verificamos que os mesmos ocorrem a direita e à esquerda, concomitantemente, em 2 conjuntos, representados pelas abservacões de numeros 10 e 26; o primeiro deles apresenta o ureter colocado sobre a linha craniocaudal, entre os quadrantes caudolateral e caudomedial e, a outro, sobre a linha mediolateral, entre os quadrantes craniolateral e caudolateral. Nos demais, nao observamos correspondência entre os antimeros, ficando, nestes casos, o ureter do lado oposto, no quadrante caudomedial, como aliás acontece no agrupamento maior (22 vezes $-73,3 \% \pm 8,1)$ de pares de ings.

\section{COMENTRRIOS}

Como já foi adiantado, o presente estudo sobre o local de emergência do ureter, em bovinos da raca Hereford. soma-se a trabalho anterior (TEIXEIRR FILHO \& FERNANDES FILHO, 4, 1982) relacionado a analise da posicáo das arterias e veias na regiao hilar do rim, colocados dentro do objetivo mais amplo de examinar o comportamento dos integrantes do pediculo renal e a vascularizaça dos órgasos, com vistas, nao apenas, ao conhecimento mais preciso da anatomia dos animais domesticos, mas tambem, o melhor enfoque de aspectos comparativos e mesmo pecutiaridades raciais.

Os tratadistas classicos, cabe reLembrar que, sempre se limitam a registrar a presenca do ureter emergindo pelo hilo, sem detalhes mais precisos, nao so ao cuidar especificamente dos bovinos, cOMO MARTIN \& SCHAUDER, 2 (1938); MONTANE \& BOURDELLE, 3 (1917) mas tambem para qualquer outra especie; tal fato, e de ressaltar, sugere-nos a necessidade de rever o assunto em outros animais domesticas nos quais, frequentemente, convenciona-se a ponto de emergência desse ducto, coincidindo camo centro geometrico da regiăo examinada. Sobre os nossos resultados convem. de inicio, realcar que o ureter foi Localizado no quadrante caudolateral na maior parte das pecas, tanto a direite $(90,0 \%)$ como à esquerda $(73,3 \%)$, coincidindo com a representacaro mais comum anotada nos esquemas apresentados por MARIRNA \& BORELLI, 1 (1986), dara os zebulnos da raca Nelore. Por outra parte, observamos ainda, quanto as variacơes de posiça, maior incidencia no rim esquerdo $(26,7 \%)$ do que no direito $(10,0 \%)$, justificada, a nosso ver, pelas caracteristicas que o orga apresenta desse lado, nos ruminantes; de fato, íace à presenca do rumen e suas constantes e nitidas modificaças de volume, o rim esquerdo apresenta-se maior e aeslocado para a direita, mostrando o pedlculo nitidamente mais longo de farma a permitir maior mobilidade para assim, Doder acompanhar as referidas mudancas de volume daquele diverticulo gastrico.

A assertiva ganha reforco ao ouse varmos identica conotaça nas ilustracões de MARIANA \& BORELLI, 1 (1985) e, acrescente-se, com o acentuado paralalismo de ocorrencia. Por outro lacio, encontramos, ocasionalmente, colocacares nao registradas pelos citados Ah. sendo - contrario, também valido. Para melnor ilustrar tais comparaças, optamos dor expô-las, seja no tocante ao local de emergencia, seja quanto à sua frequencia percentual, de forma resumida, no Ouad. 1. 

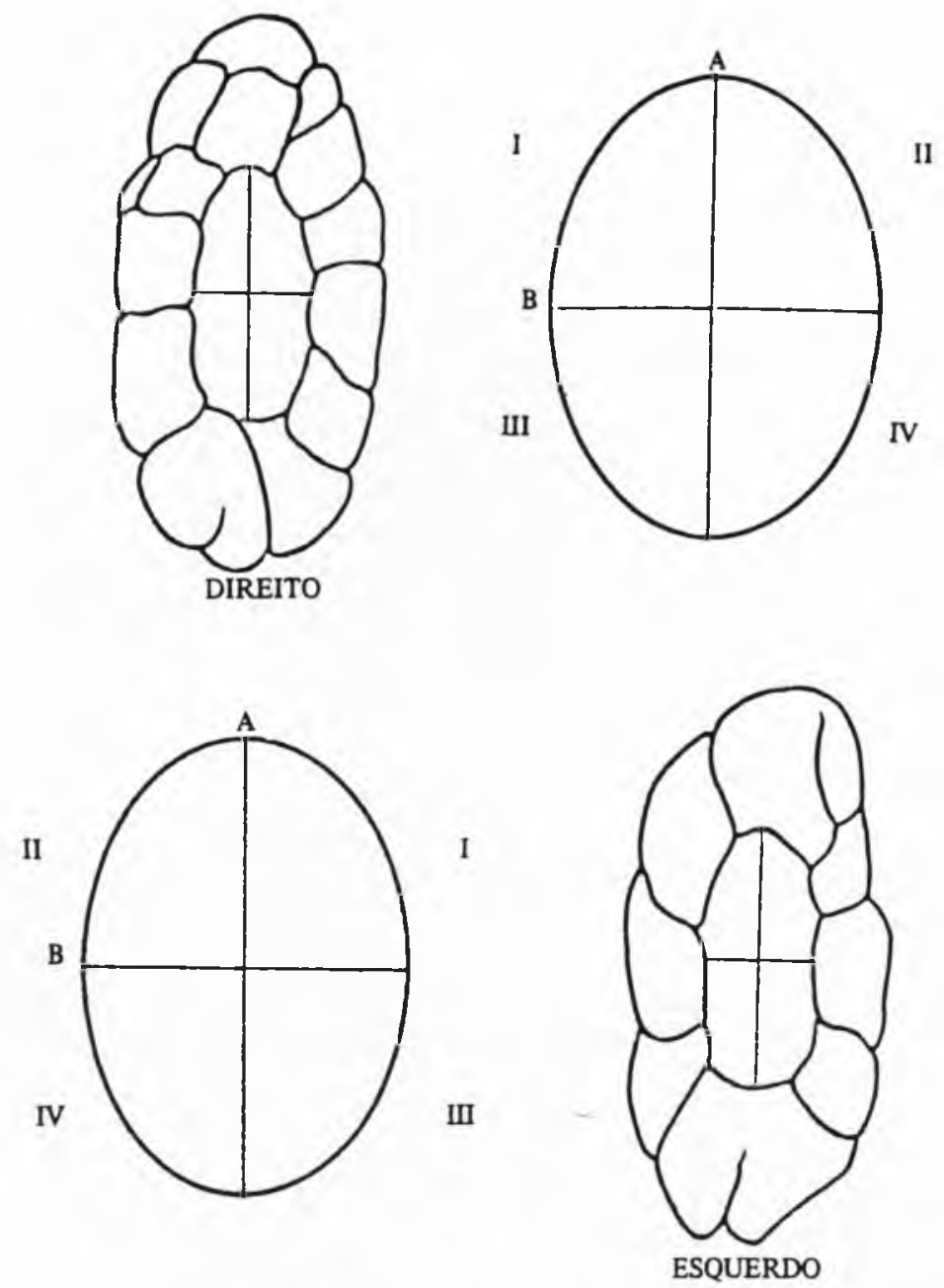

FIGURA 1 - Representacão semi-esquematica dos rins direita e esquerdo de bovinos da raca Hereford mostrando o hilo dividido em quadrantes pelo tracado de duas linhas perpendiculares, indicados nominalmente, nos desenhos esquematicos colocados ao lado e que serviram de base para andises e ilustractas.

A. Linha craniocaudal

B. Linha Laterolateral

I. Quadrante craniolaterab

II. Uuadrante craniomedial

II I. Quadrante caudolaterab

IV. Quadrante caudomedial 
Sobre a posição do ureter no hilo renal de bovinos da raça Hereford.
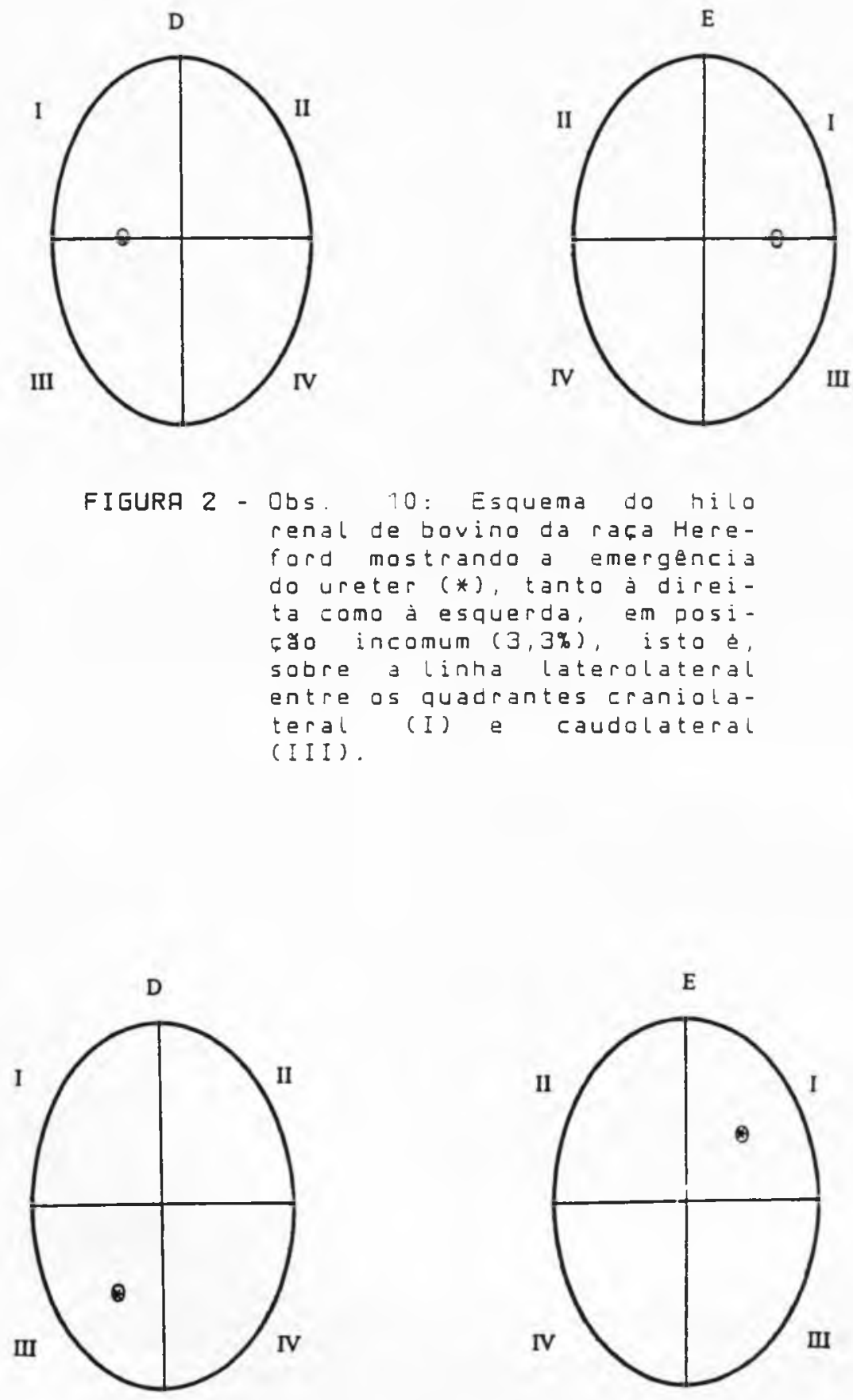

FIGURA 3 - Obs. 14: Esquema do hilo renal de bovino da raça Hereford mostrando, a direita, a localizaço mais frequente $(90,0 \%)$ da emergência do ureter (*), isto e, no quadrante caudolateral (III) e, a esquerda em posicao incomum $(3,3 \%)$ ou seja, no quadrante craniolateral (I). 


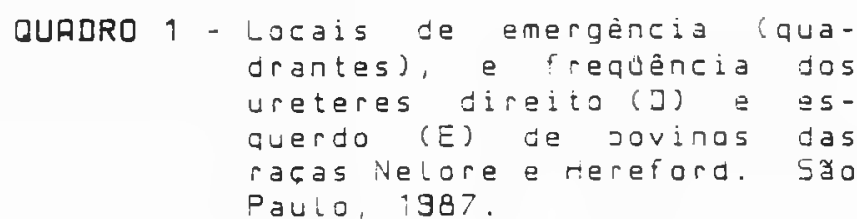

RACA
de

IEIXEIPA FILHO, A.; FERNANDES FILHO, PEDUTI NETO, J. POSition of the ureter in the renal hilus of Hereford cattle. Rev. Fac. Med. Vet. Zootec. Univ. 5. Paulo, 25(2):215-219, 1988.

SUMMRRY: There was studied the position of the emergence of the ureter in the hilus in 30 pairs of kidneys of Here- ford cattle. For that, the hilus was divided in quadrants so named: Craniolateralis, craniomedialis, caudolateralis and caudomedialis. The results ootained show different sites of emergence, especially in the left kidney.

UNITERMS: Anatomy of cattle; Urogenital system; Kidney; Ureter

REFERENCIAS BIBLIOGRAFICAS

\section{MARIANA, A. \& BORELLI, V. Contri- buica ao estudo dos elementos vasculares, arteriais e venosos, do hilo renal, em bovinos da raca Nelore. In: SEMANA DE MEDI- CINA VETERINARIA DA FACULDADE DE MEDICINA VETERINARIA E ZOOTECNIA DA USP, 4. , ENCONTRO NACIONAL DE PROFESSORES DE INSPECAO, 2., ENCONTRO NACIONAL DE PRDFESSDRES DE ZOOTELNIA, 1., Sgo Paulo, 1986. Resumos. p. 40 .}

2 - MARTIN, P. \& SCHAUDER, W. Lehrbuch der Anatomie der Haustiere. Stuttgart, Schickardt \& Ebner, 1938. v. 3, t. 3, p.277-278.
3 - MONTANE, L. \& bourdelle, E. Anatomie regionale des animaux domes. tiques. Paris, Libraire J.-B. 日aillière, 1917. v. 2, 0.300.

4 - TEIXEIRA FILHO, A. \& FERNANDES FILHO, A. Contribuicao ao estudo dos elementos vasculares, arteriais e venosos, do hilo renal, em bovinos da raca Hereford. In: SEMANA DE VETERINARIA DA FACUL DADE DE MEDICINA VETERINARIA E ZOOTECNIA DA USP, 1., S30 PaULO, 1982. Resumos. p. 31. 